\title{
Idosos em uso de terapia nutricional internados para reabilitação: perfil, avaliação da oferta calórica/proteica e desmame de dietas enterais
}

\section{Elderly people using nutricional terapy hospitalized for rehabilitation: profile, evaluation of caloric and protein supply and weaning of enteral diets}

Personas mayores en uso de terapia nutricional enteral para la rehabilitación: perfil, evaluación de la oferta calórica / proteína y desteste de las dietas enterales

Rafael Alves Mata de Oliveira ${ }^{1}$

Yulle Fourny Barão ${ }^{2}$ Maruska Dias Soares ${ }^{3}$ Fernanda Zanoni Cônsolo ${ }^{4}$

\footnotetext{
${ }^{1}$ Especialista em Cuidados Continuados Integrados: Atenção à Saúde do Idoso, e bacharel em Nutrição pela Universidade Federal de Mato Grosso do Sul (UFMS). E-mail: nutrirafaamo@gmail.com, ORCID: http://orcid.org/0000-0002-0645-7461
}

2 Especialista em Cuidados Continuados Integrados: Atenção à Saúde do Idoso, e bacharel em Nutrição pela Universidade Federal de Mato Grosso do Sul (UFMS).E-mail: yullefourny@hotmail.com, ORCID: http://orcid.org/0000-0001-5591-7893

${ }^{3}$ Mestre em Saúde Pública e Nutricionista pela Universidade Estadual do Ceará. Docente do curso de Nutrição da Faculdade de Ciências Farmacêuticas, Alimentos e Nutrição

da Universidade Federal de Mato Grosso do Sul (UFMS). Docente da Residência Multiprofissional em Cuidados Continuados Integrados da UFMS/Hospital São Julião. E-mail: maruska.diass@gmail.com, ORCID: http://orcid.org/0000-0003-2554-1543

${ }^{4}$ Doutora em Nutrição pela Universidade Federal de Mato Grosso do Sul (UFMS). Professora do Curso de Nutrição da UFMS. E-mail: fernandazanoni@yahoo.com.br, ORCID: http://orcid.org/0000-0002-0812-2817 
Resumo: Objetivou-se identificar o perfil nutricional de idosos em uso de Terapia Nutricional Enteral (TNE), por sondas ou ostomias, em reabilitação em uma Unidade de Cuidados Continuados Integrados, e sua evolução ou não para o desmame. Trata-se de um estudo transversal, descritivo e longitudinal, com amostra de 62 idosos, através de coleta de dados secundários em prontuários. Houve busca das seguintes informações: dieta (tempo de uso de TNE, valor energético e proteico); dados antropométricos (peso, altura, Índice de Massa Corporal [IMC], circunferência do braço); e diagnóstico nutricional na admissão e alta. Conclui-se a alta prevalência de desmame de TNE (81,3\%); que a média da oferta do valor energético e proteico das dietas não estava adequada segundo as principais recomendações; e que os pacientes, em média, mantiveram o seu estado nutricional durante a internação.

Palavras-chave: idoso; nutrição enteral; reabilitação.

\begin{abstract}
This study aimed to identify the nutritional profile of elderly people using Enteral Nutritional Therapy (ENT), by enteral tubes or ostomies, undergoing rehabilitation in an Integrated Continuing Care Unit, and whether or not they progress to weaning. It is a cross-sectional, descriptive, and longitudinal study, with a sample of 62 elderly, through secondary data collection in medical records. The following information was sought: diet (time of use of ENT, energy and protein value); anthropometric data (weight, height, body mass index [BMI], arm circumference); nutritional diagnosis at admission and discharge. It is concluded the high prevalence of ENT weaning (81,3\%), that the mean dietary energy and protein value was not adequate according to the main recommendations, and that the patients maintained their nutritional status during hospitalization.
\end{abstract}

Keywords: aged; enteral nutrition; rehabilitation.

Resumen: El objetivo de este estudio fue identificar el perfil nutricional de las personas de edad avanzada que usan terapia enteral nutricional (TEN) mediante sondas u ostomías, que se someten a rehabilitación en una Unidad Integrada de Atención Continua y si progresan o no al destete. Este es un estudio transversal, descriptivo y longitudinal, con una muestra de 62 ancianos, a través de la recolección de datos secundarios en registros médicos. Se buscó la siguiente información: dieta (tiempo de uso de TEN, valor energético y proteico); datos antropométricos (peso, altura, índice de masa corporal [IMC], circunferencia del brazo); diagnóstico nutricional al ingreso y al alta. Se concluye la alta prevalencia de destete de TEN (81,3\%); que el valor medio de energía y proteína de la dieta no fue adecuado de acuerdo con las recomendaciones principales; y que los pacientes mantuvieron su estado nutricional durante la hospitalización.

Palabras clave: anciano; nutrición enteral; rehabilitación. 


\section{INTRODUÇÃO}

O envelhecimento populacional está crescendo rapidamente em todo o mundo, superando outras faixas etárias. Os idosos se caracterizam por ser um grupo vulnerável em razão do processo fisiológico de envelhecimento, o que acarreta maiores custos aos serviços de saúde. As internações em unidades hospitalares são mais frequentes e a duração da ocupação dos leitos é maior em relação à população mais jovem, perdurando por muito tempo e exigindo, assim, acompanhamento e intervenção contínua.

Existe uma forte correlação entre idade e dependência, sendo que a prevalência de indivíduos com limitações se amplia conforme consideramos grupos etários com maior idade. Sobretudo, os idosos exigem um tratamento contínuo em termo de apoio e cuidados.

Diante disso, necessita-se de unidades e modelos hospitalares preparados para atender essa população, como as Unidades de Cuidados Continuados Integrados (UCCI), que visam à reabilitação, readaptação e reinserção familiar e social de indivíduos com algum grau de dependência, promovendo a sua autonomia.

Sendo um grupo de maior vulnerabilidade nutricional e maior dependência no cuidado, é de grande importância planejar adequadamente as necessidades nutricionais desta população, considerando também sua patologia de base. As necessidades nutricionais dos idosos também estão relacionadas ao nível de atividade física, ao gasto calórico e suas necessidades energéticas em repouso e, além disso, à capacidade de acessar, preparar, ingerir e digerir alimentos.

Muitas vezes, devido às condições neurológicas e patológicas dos idosos, este grupo torna-se inapto ao consumo alimentar exclusivamente pela via oral, necessitando de dispositivos de alimentação, como as sondas enterais e as ostomias, de forma total ou parcial, para atingir as suas necessidades nutricionais. A Terapia Nutricional Enteral (TNE) é o conjunto de procedimentos terapêuticos que objetivam manter ou recuperar o estado nutricional do paciente, através da nutrição enteral, que é considerada um alimento para fins especiais, podendo ser utilizada de forma isolada ou para complementar a alimentação oral. 
Considerando estas evidências, é possível observar a tendência do aumento da população idosa e a forte relação entre a desnutrição, o avançar da idade e a importância das UCCI no apoio à recuperação e manutenção da saúde dos idosos.

Tendo em vista a grande relevância da nutrição adequada em pacientes idosos em uso de Terapia Nutricional Enteral, o objetivo do presente trabalho foi identificar o perfil nutricional dessa população e o principal desfecho em relação ao desmame dessa via de alimentação em um hospital de retaguarda.

\section{METODOLOGIA}

Trata-se de um estudo transversal, descritivo e longitudinal, em uma Unidade de Cuidados Continuados Integrados, de um hospital de retaguarda, a partir da coleta de dados secundários, entre janeiro de 2017 e janeiro de 2019. Conforme protocolo da instituição, o tempo de internação gira em torno de 15 dias a 60 dias, dependendo de cada caso clínico avaliado, embora existam exceções ao prazo.

A amostra foi composta por idosos de ambos os sexos, com idade igual ou superior a 60 anos que fizeram uso da nutrição enteral (por sondas ou ostomias) em algum momento da internação. Foram excluídos da amostra os idosos com alimentação por via oral de forma exclusiva. A fim de evitar viés na pesquisa, os pacientes com associação da via oral à enteral foram excluídos do estudo.

Para a caracterização da amostra, foram consideradas as seguintes informações: idade; dias de internação; diagnóstico principal e comorbidades. Em relação à dieta enteral ofertada, foram coletados: o tempo de uso da nutrição enteral; calorias ofertadas (kcal/kg/dia); proteínas (g/kg/ dia) (considerando o peso atual aferido ou estimado); volume da dieta; características e densidade calórica da fórmula.

Para a caracterização da avaliação nutricional da amostra, foram coletados os dados de admissão e alta, a saber: peso atual (aferido ou estimado); altura (referida, aferida ou estimada); Índice de Massa Corporal (IMC), segundo a referência Lipschitz (1994) para idosos (Tabela 1); Circunferência 
do Braço (CB) por percentis, por Frisancho (1981); e adequação da CB, segundo Blackburn e Thornton (1979) (Tabela 2).

Tabela 1 - Classificação de Índice de Massa Corporal (IMC) para idosos, segundo Lipschitz (1994)

\begin{tabular}{c|c}
\hline Referência & Classificação \\
\hline$<22 \mathrm{~kg} / \mathrm{m}^{2}$ & Magreza \\
\hline $22 \mathrm{~kg} / \mathrm{m}^{2}$ a $27 \mathrm{~kg} / \mathrm{m}^{2}$ & Eutrofia \\
\hline$>27 \mathrm{~kg} / \mathrm{m}^{2}$ & Sobrepeso \\
\hline
\end{tabular}

Fonte: Lipschitz (1994).

Tabela 2 - Classificação da Adequação da Circunferência do Braço (\%), segundo Blackburn e Thornton (1979)

\begin{tabular}{c|c}
\hline Referência & Classificação \\
\hline$<70 \%$ & Desnutrição grave \\
\hline $70 \%$ a $80 \%$ & Desnutrição moderada \\
\hline $80 \%$ a $90 \%$ & Desnutrição leve \\
\hline $90 \%$ a $110 \%$ & Eutrofia \\
\hline $110 \%$ a $120 \%$ & Sobrepeso \\
\hline$>120 \%$ & Obesidade \\
\hline
\end{tabular}

Fonte: Blackburn e Thornton (1979).

Além disso, foi investigado se os idosos conseguiram realizar o desmame de nutrição enteral na internação, se houve associação de dieta enteral com a via oral e o seu desfecho clínico (alta hospitalar, transferência de setor ou óbito).

A comparação da quantidade de proteína, por peso corporal, por dia, ofertada aos pacientes na dieta enteral, com aquela preconizada pela literatura, foi realizada por meio do teste t-student de uma amostra. O mesmo teste foi utilizado na comparação da quantidade de calorias, por peso corporal, por dia, ofertada aos pacientes na dieta enteral, com aquela preconizada pela literatura. Tanto a média de proteínas quanto a de calorias foram comparadas com as recomendações da European Society for Clinical Nutrition and Metabolism (ESPEN, 2018) e da Sociedade Brasileira de Nutrição Parenteral 
e Enteral (BRASPEN, 2019). Já a comparação entre a admissão e a alta hospitalar, em relação às variáveis peso, IMC e adequação da $\mathrm{CB}$, foi realizada por meio do teste t-student. A avaliação da associação entre o diagnóstico nutricional na admissão e aquele na alta hospitalar foi realizada por meio do teste de McNemar. O mesmo teste foi utilizado na avaliação da associação entre o momento da admissão e aquele da alta, em relação à variável perda muscular. A análise estatística foi realizada por meio do programa estatístico SPSS, versão 23.0, considerando um nível de significância de $5 \%$.

O presente estudo foi aprovado pelo Comitê de Ética em Pesquisas da Universidade Federal de Mato Grosso do Sul (Parecer: 3.096.734). Para a coleta de dados, foi disponibilizado o Termo de Consentimento do Uso de Banco de Dados (TCUD).

\section{RESULTADOS E DISCUSSÃO}

A amostra foi constituída por 62 idosos. A idade variou entre 60 e 95

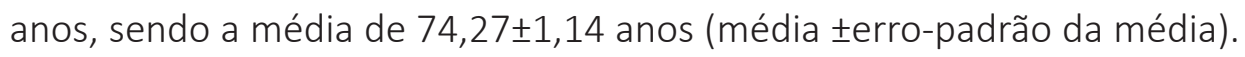
O diagnóstico principal da maioria dos pacientes foi acidente vascular encefálico (AVE) $(79,03 \%(n=49))$, sendo a hipertensão a comorbidade mais observada $(83,87 \%(n=52))$; seguida de diabetes mellitus, presente em $40,32 \%(n=25)$ dos casos. Em relação à nutrição enteral, o tempo médio de uso foi de $18,62 \pm 2,68$ dias, sendo que o uso de nutrição enteral exclusiva foi de $16,13 \pm 2,52$ dias. Vale ressaltar que os pacientes ficam de quinze a sessenta dias internados no hospital do estudo.

Menezes e Forte (2019), em um estudo observacional, do tipo coorte retrospectivo e analítico, com pacientes idosos em uso de nutrição enteral, com média de idade de $76 \pm 10,12$ anos, observaram que a principal morbidade entre a amostra foi o AVE, representando 31,2\% dos diagnósticos. Tais achados corroboram com o presente estudo, em que a patologia mais prevalente também foi o AVE.

Pacientes idosos que tiveram AVE podem ser beneficiados e protegidos contra a desnutrição, a partir de uma avaliação nutricional individualizada, dando atenção especial à ingestão alimentar e evitando a perda de peso (HA et al., 2010). 
Estudo realizado na cidade de Ouro Preto, MG, avaliou também a população idosa e encontrou os seguintes resultados com relação às doenças crônicas: $82 \%$ dos idosos foram diagnosticados com hipertensão arterial, enquanto 25\% apresentavam diabetes (PREVIATO et al., 2015). Embora o presente estudo abranja apenas idosos hospitalizados, verificamos similaridade em relação ao alto índice de comorbidades, como a hipertensão e o diabetes mellitus, nas duas populações estudadas.

Nas Tabelas 3 e 4, respectivamente, estão apresentadas as caracterizações dos participantes do estudo de acordo com a idade, diagnóstico principal, comorbidades, tempo de internação, desfecho clínico, aspectos da nutrição enteral e desmame.

Tabela 3 - Caracterização dos participantes do estudo de acordo com a idade, diagnóstico principal, comorbidades, tempo de internação e desfecho clínico

\begin{tabular}{|c|c|}
\hline Variável & Média \pm EPM ou \% (n) \\
\hline Idade (60 anos a 95 anos) & $74,27 \pm 1,14$ \\
\hline \multicolumn{2}{|l|}{ Diagnóstico principal } \\
\hline AVC & $79,03(49)$ \\
\hline Doenças respiratórias & $6,45(4)$ \\
\hline Outras & $14,52(9)$ \\
\hline \multicolumn{2}{|l|}{ Comorbidades } \\
\hline Hipertensão & $83,87(52)$ \\
\hline Diabetes & $40,32(25)$ \\
\hline Outras patologias & $38,7(24)$ \\
\hline Tempo de internação (7 dias a 77 dias) & $37,33 \pm 1,77$ \\
\hline \multicolumn{2}{|l|}{ Desfecho clínico } \\
\hline Alta hospitalar & $90,32(56)$ \\
\hline Transferência de setor & $9,68(6)$ \\
\hline
\end{tabular}

Fonte: Elaborado pelos próprios autores. 
Tabela 4 - Caracterização dos participantes do estudo de acordo com aspectos da nutrição enteral e desmame

\begin{tabular}{|c|c|}
\hline Variável & Média \pm EPM ou $\%$ (n) \\
\hline \multicolumn{2}{|l|}{ Nutrição enteral } \\
\hline Uso de nutrição enteral exclusiva (1 a 66 dias) & $16,13 \pm 2,52$ \\
\hline Calorias de nutrição enteral exclusiva (kcal) & $1230,92 \pm 49,93$ \\
\hline $\begin{array}{l}\text { Proteína/peso corporal de nutrição enteral } \\
\text { exclusiva (g/kg/dia) }\end{array}$ & $0,72 \pm 0,04$ \\
\hline $\begin{array}{l}\text { Kcal/peso corporal de nutrição enteral exclusiva } \\
\text { (kcal/kg/dia) }\end{array}$ & $19,55 \pm 0,94$ \\
\hline Volume da dieta de nutrição enteral exclusiva (mL) & $1.013,14 \pm 39,06$ \\
\hline \multicolumn{2}{|l|}{ Desmame da nutrição enteral durante a internação } \\
\hline Não & $18,8(12)$ \\
\hline Sim & $81,3(52)$ \\
\hline Sem informação & 1 \\
\hline
\end{tabular}

Fonte: Elaborado pelos próprios autores.

No presente estudo, as dietas variaram, em termos de calorias, sendo a quantidade de calorias média de 1230,92×49,93 por paciente. Já a quantidade média de proteína foi de $0,81 \mathrm{~g} / \mathrm{kg} / \mathrm{dia}$, e, em relação à quantidade de calorias por peso corporal, por dia, sua quantidade média foi de 19,74. A média da oferta de proteína e de caloria, bem como sua comparação com os valores preconizados, está descrita na Tabela 5.

Tabela 5 - Comparação entre a oferta calórica e proteica obtida na pesquisa e os valores preconizados pela ESPEN, com o valor de " $p$ "

\begin{tabular}{lccc}
\hline & Preconizado & $\begin{array}{c}\text { Valor obtido na } \\
\text { pesquisa }\end{array}$ & Valor de "p" \\
\hline Kcal & $30 \mathrm{kcal} / \mathrm{kg} / \mathrm{dia}$ & $19,55 \pm 0,94$ & $\mathrm{p}<0,001$ \\
Proteína & $1 \mathrm{~g} / \mathrm{kg} / \mathrm{dia}$ & $0,72 \pm 0,04$ & $\mathrm{p}<0,001$ \\
\hline
\end{tabular}

Fonte: Elaborado pelos próprios autores.

Segundo a ESPEN, a ingestão de proteínas em idosos deve ser de pelo menos $1 \mathrm{~g} / \mathrm{kg} /$ dia. O valor de referência da ingestão de energia em idosos é de $30 \mathrm{kcal} / \mathrm{kg} / \mathrm{dia}$, e ambas as recomendações são baseadas em 
fortes evidências científicas. A quantia deve ser ajustada individualmente em relação ao estado nutricional, nível de atividade física, status da doença e tolerância (VOLKERT et al., 2011).

Já a BRASPEN (2019) recomenda que o cálculo de necessidade energética para indivíduos idosos seja baseado em $30 \mathrm{kcal} / \mathrm{kg} / \mathrm{dia}$ a $35 \mathrm{kcal} / \mathrm{kg} /$ dia, enquanto a oferta proteica dos idosos deve variar entre 1,0 g a 1,5 g de proteínas por kg de peso corporal por dia.

A recomendação tradicional para ingestão de proteínas de $0,8 \mathrm{~g} / \mathrm{kg}$ de peso corporal por dia para adultos de todas as idades está atualmente sob discussão para idosos, com base em evidências crescentes de pesquisas experimentais e epidemiológicas de que os idosos precisam de quantidades maiores de proteína para a preservação ideal da massa magra corporal, funções corporais e saúde. Quantidades diárias de 1,0 g/kg a 1,2 g/kg de peso corporal foram sugeridos para idosos saudáveis por vários grupos de especialistas (DEUTZ et al., 2014).

Em relação à fórmula industrializada de nutrição enteral dos pacientes do presente estudo, a mais utilizada foi a polimérica, normocalórica e normoproteica, com densidade calórica de 1,2 kcal/ml, com 56,45\% dos idosos utilizando essa dieta, sendo que a maioria dos pacientes idosos utilizou a sonda nasogástrica, comparada com a sonda nasoenteral e as ostomias.

Estudo recente apontou que a maioria dos idosos em uso de nutrição enteral $(95,4 \%)$ se beneficiaram de uma fórmula polimérica, nutricionalmente completa, com densidade calórica entre 1,0 kcal/ml e 1,2 kcal/ml, normocalórica e normoproteica (MENEZES; FORTES, 2019).

Na Tabela 6, estão apresentados os resultados da comparação entre a admissão e a alta hospitalar, em relação às variáveis peso, IMC, circunferência do braço e adequação da circunferência do braço. Não houve uma diferença significativa entre o momento da admissão e aquele da alta, em relação a nenhuma destas variáveis (teste t-student pareado, valor de $p$ variando entre 0,626 e 0,890). 
Tabela 6 - Resultados da comparação entre a admissão e a alta hospitalar, em relação às variáveis peso, IMC e adequação da circunferência do braço

\begin{tabular}{|c|c|c|c|}
\hline \multirow{2}{*}{ Variável } & \multicolumn{2}{|c|}{ Momento } & \multirow{2}{*}{$\begin{array}{c}\text { Valor de } \\
\mathbf{p}\end{array}$} \\
\hline & Admissão & Alta & \\
\hline Peso (kg) & $61,58 \pm 2,27$ & $61,51 \pm 2,34$ & 0,947 \\
\hline $\mathrm{IMC}\left(\mathrm{kg} / \mathrm{m}^{2}\right)$ & $23,20 \pm 0,82$ & $23,16 \pm 0,74$ & 0,914 \\
\hline Circunferência do braço (cm) & $26,42 \pm 1,09$ & $26,31 \pm 0,91$ & 0,756 \\
\hline Adequação da circunf. do braço (\%) & $87,12 \pm 2,84$ & $86,56 \pm 2,55$ & 0,636 \\
\hline
\end{tabular}

Os resultados estão apresentados em médiaterro-padrão da média. Valor de $p$ no teste t-student pareado.

Fonte: Elaborado pelos próprios autores.

Não houve associação significativa entre o diagnóstico na admissão e aquele na alta hospitalar, tanto para classificação pelo IMC (teste de McNemar, $p=0,513$ ) como pela classificação de acordo com a adequação da circunferência do braço $(p=0,506)$ e pelo diagnóstico nutricional $(p=0,223)$. Em relação à comparação do IMC na admissão e da alta, não houve associação estatística significativa ( $p=0,513)$.

Os resultados da avaliação da associação entre o momento da admissão e aquele da alta, em relação à variável adequação de CB (\%), demonstraram que em nenhuma delas houve uma mudança no perfil destas variáveis, entre a admissão e a alta hospitalar (teste de McNemar, valor de p variando entre 0,625 e 1,000).

Em relação à comparação entre o IMC na admissão e na alta, não houve diferença estatística significativa $(p=0,860)$. Em porcentagem, temos os seguintes diagnósticos: eutrofia $(54,17 \%$; $n=13)$; magreza $(29,17 \%$; $n=7)$ e sobrepeso $(16,66 \% ; n=4)$.

Outro estudo com a população na mesma faixa etária apontou também que não foram observadas diferenças estatísticas entre idade, peso e IMC nos quatro períodos diferentes de avaliação $(p>0,05)$, com o IMC médio de $21,04 \pm 4,24 \mathrm{~kg} / \mathrm{m}^{2}$ e com o diagnóstico nutricional mais prevalente à desnutrição (65,1\%) (MENEZES; FORTES, 2019).

Em estudo brasileiro, com resultados de acordo com IMC, 53,6\% dos idosos foram classificados com sobrepeso, 35,7\% apresentavam-se eutró- 
ficos e 10,7\% foram diagnosticados com magreza, sem diferença estatística entre os sexos. Todavia a prevalência de excesso de peso (60\%) foi maior entre as mulheres, enquanto no grupo dos homens houve maior prevalência de eutrofia (62,5\%) (PREVIATO et al., 2015).

Taxas de prevalência de desnutrição relatadas dependem muito da definição usada, mas geralmente estão abaixo de $10 \%$ entre pessoas idosas independentes e aumentando até dois terços de pacientes idosos em hospitais de cuidados agudos e reabilitação (CEREDA et al., 2016).

No diagnóstico nutricional, também não houve associação (teste de McNemar, $p=0,223$ ), entre os momentos de análise em relação a este diagnóstico, na admissão e na alta, mantendo o estado nutricional durante a internação.

Estudo que comparou a dieta via oral e a enteral em idosos institucionalizados na Alemanha apontou que pacientes com nutrição enteral são marcadamente mais afetados pela desnutrição do que aqueles que se alimentam por via oral. Por outro lado, os dados demonstraram que a massa muscular pode ser mantida ou alcançada através da alimentação por sondas ou ostomias, indicada pelas medidas do Índice de Massa Corporal (IMC) e a circunferência do braço (VOLKERT et al., 2011), o que corrobora nosso estudo, já que os idosos mantiveram seu estado nutricional durante a internação, comparando o Índice de Massa Corporal e a Circunferência do Braço da admissão e da alta.

Em relação ao desmame de nutrição enteral, a maioria $(81,3 \%-n=52)$ conseguiu esse feito durante a internação, sendo que apenas $18,7 \%$ (n-12) foram de alta ou transferidos de setor com via enteral exclusiva ou mista como via de alimentação. Finalmente, o desfecho da maior parte dos pacientes avaliados neste estudo foi a alta hospitalar (89,1\% - n=57).

Estudo europeu realizado com o objetivo de desenvolver um modelo preditivo simples do resultado da disfagia em pacientes com AVE, com média de idade de 75 anos (de 50 a 94 anos), em um hospital de reabilitação, com todos os pacientes na admissão com nutrição enteral por sondas ou ostomias, com uma amostra de 30 pacientes, teve os seguintes resultados: 12 indivíduos foram desmamados da nutrição enteral e 18 mantiveram a nutrição enteral de forma exclusiva, ou seja, uma prevalência de desmame 
de $40 \%$ em relação ao universo amostral (OTO et al., 2009), logo o nosso estudo teve uma alta prevalência de desmame de nutrição enteral, em consonância com o objetivo da reabilitação nutricional das Unidades de Cuidados Continuados Integrados.

A despeito dos resultados encontrados neste estudo, a ausência de dados em prontuários compõe uma grande limitação, o que justifica a necessidade de maior empenho dos profissionais que atendem idosos no âmbito hospitalar e reabilitação sobre a importância de informações completas, como também avaliação mais precisa na admissão e alta, priorizando a segurança do paciente e subsidiando estudos futuros nessa população tão fragilizada.

\section{CONCLUSÃO}

Grande parte dos idosos conseguiu concluir o desmame da Terapia Nutricional Enteral (TNE) com, em média, um mês de internação. Em relação à antropometria, os pacientes (maioria) mantiveram o seu estado nutricional durante toda a internação. Houve proporção maior de indivíduos desnutridos do que com sobrepeso, o que é frequente entre os idosos hospitalizados, contrapondo a alta prevalência de idosos com sobrepeso na comunidade. Em relação à dieta, os pacientes estavam recebendo menor quantidade de calorias e proteínas do que as recomendações, o que contribui para o aumento da desnutrição hospitalar.

Este estudo demonstra a importância das Unidades de Cuidados Continuados Integrados na reabilitação de pacientes idosos com vias alternativas de alimentação, reestabelecendo a independência e autonomia em suas práticas alimentares. É de grande relevância que sejam realizados e aprofundados mais estudos sobre a utilização de terapia nutricional enteral em idosos, bem como seu desmame, estejam eles internados ou em domicílio, para que sejam mais efetivas as ações dos nutricionistas em relação à reabilitação desses pacientes. 
Idosos em uso de terapia nutricional internados para reabilitação: perfil, avaliação da oferta calórica/proteica e desmame de dietas enterais

\section{REFERÊNCIAS}

BLACKBURN, G. L.; THORNTON, P. A. Nutritional assessment of the hospitalized patient. The Medical clinics of North America, [s.I.], v. 63, n. 5, p. 11103-15, 1979.

BURGOS, R.; BRÉTON, I.; CEREDA, E.; DESPORT, J. C.; DZIEWAS, R.; GENTON, L.; GOMES, F.; JÉSUS, P.; LEISCHKER,A.; MUSCARITOLI, M.; POULIA, K. A.; PREISER, J. C.; VAN DER MARCK, M.; WIRTH, R.; SINGER, P.; BISCHOFF, S. C . ESPEN guideline clinical nutrition in neurology. Clinical Nutrition, [s.I.], v. 37, n. 1, p. 354-96, 2018.

CEREDA, E.; PEDROLLI, C.; KLERSY, C.; BONARDI, C.; QUARLERI, L.; CAPELLO, S.; TURRI, A.; RONDANELLI, M.; CACCIALANZA, R. Nutritional status in older persons according to healthcare setting: a systematic review and meta-analysis of prevalence data using MNA $^{\circledast}$. Clinical Nutrition, [s.l.], v. 35, n. 6, p. 1282-90, 2016.

DEUTZ, N. E. P; BAUER, J. M.; BARAZZONI, R.; BIOLO, G.; BOIRIE, T.; BOSY-WESTPHAL, A.; CEDERHOLM, T.; CRUZ-JENTOFT, A.; KRZNARIÇ, Z.; NAIR, K. S.; SINGER, P.; TETA, D.; TIPTON, K.; CALDER, P.C. Protein intake and exercise for optimal muscle function with aging: recommendations from the ESPEN Expert Group. Clinical Nutrition, [s.l.], v. 33, n. 6, p. 929-36, 2014.

FRISANCHO, A. R. New norms of upper limb fat and muscle areas for assessment of nutritional status. The American Journal of Clinical Nutrition, [s.I.], v. 34, n. 11, p. 2540-5, 1981.

GONÇALVES, T. J. M.; HORIE, L. M.; GONÇALVES, S. E. A. B.; BACCHI, M. K.; BAILER, M. C.; BARBOSA, T. G. Diretriz BRASPEN de terapia nutricional no envelhecimento. Braspen J, [s.I.], v. 34, n. 2, p. 2-58, 2019.

HA, L.; HAUGE, T.; SPENNING, A. B.; IVERSEN, P. O. Individual, nutritional support prevents undernutrition, increases muscle strength and improves QoL among elderly at nutritional risk hospitalized for acute stroke: a randomized, controlled trial. Clinical Nutrition, [s.l.], v. 29, n. 5, p. 567-73, 2010.

LIPSCHITZ, D. A . Screening for nutritional status in the elderly. Primary Care: Clinics in Office Practice, [s.I.], v. 21, n. 1, p. 55-67, 1994.

MENEZES, C. S.; FORTES, R. C. Nutritional status and clinical evolution of the elderly in home enteral nutritional therapy: a retrospective cohort study. Revista latinoamericana de enfermagem, São Paulo, v. 27, p. 397-403, 2019.

OTO, T.; KANDORI, Y.; OHTA, T.; DOMEN, K.; KOYAMA, T. Predicting the chance of weaning dysphagic stroke patients from enteral nutrition: a multivariate logistic 
modelling study. European journal of physical and rehabilitation medicine, [s.I.], v. 45, n. 3, p. 355-62, 2009.

PREVIATO, H. D. R. A.; BARROS, F. S. S.; MELLO, J. B. M.; SILVA, F. C. S.; NIMER, M. Perfil clínico-nutricional e consumo alimentar de idosos do Programa Terceira Idade, Ouro Preto-MG. DEMETRA - Alimentação, Nutrição \& Saúde, Rio de Janeiro, v. 10, n. 2, p. 375-88, 2015.

SOCIEDADE BRASILEIRA DE NUTRIÇÃO; PARENTERAL E ENTERAL; COLÉGIO BRASILEIRO DE CIRURGIÕES; SOCIEDADE BRASILEIRA DE CLÍNICA MÉDICA; ASSOCIAÇÃO BRASILEIRA DE NUTROLOGIA. Terapia nutricional para pacientes na senescência (Geriatria). Participantes: Celano, R. M. G., Loss, S. H., Negrão, R. J. N. Projeto Diretrizes - Associação Médica Brasileira e Conselho Federal de Medicina, São Paulo, 2011. Disponível em: https://diretrizes.amb.org.br/_BibliotecaAntiga/ terapia_nutricional_para_pacientes_na_senescencia_geriatria.pdf. Acesso em: 10 out. 2020.

VOLKERT, D.; BECK, A. M.; CEDERHOLM, T.; CRUZ-JENTOFT, A.; HOOPER, L.; KIESSWETTER, E.; MAGGIO, M.; RAYNARD-SIMON, A.; SIEBER, C. C.; SOBOTKA, L.; BISCHOFF, S. C. ESPEN guideline on clinical nutrition and hydration in geriatrics. Clinical Nutrition, [s.I.], v. 38, n. 1, p. 10-47, 2018.

VOLKERT, D.; PAULY, L.; STEHLE, P.; SIEBER, C. C. Prevalence of malnutrition in orally and tube-fed elderly nursing home residents in Germany and its relation to health complaints and dietary intake. Gastroenterology Research and Practice, [s.l.], v. 2011, p. 1- 9, 2011. 